\title{
Stochastic Analysis of Manpower System with Production and Sales
}

\author{
K.Hari Kumar ${ }^{1}$, P.Sekar ${ }^{2}$ \\ ${ }^{1}$ Asst.Professor, SRM University,Kattankulathur,Tamilnadu. \\ ${ }^{2}$ Principal, C.Kandaswami Naidu College, Chennai-102
}

\begin{abstract}
In this paper, Manpower System of an organization with two groups is considered. Breakdown occurs in the two groups of the Manpower System due to attrition process. Group A consists of employees other than top management level executives; group B consists of top management level executives. In this model group A is exposed to Cumulative Shortage Process (CSP) due to attrition and group B has an Erlang phase 2 distribution. Joint Laplace transform of Time to Recruit, Recruitment time and sales time has been found. Their expectations are presented with numerical illustrations.
\end{abstract}

Keywords: Manpower system, attrition, shortage, cumulative shortage process, Erlang Phase two distributions.

\section{Introduction}

In an organization, the total flow out of the Manpower System (MPS) is termed as shortage, The flow out of the MPS of an organization happens due to resignation, dismissal and death. The shortages that have occurred due to the outflow of manpower should be compensated by recruitment. But recruitment cannot be made frequently since it involves cost. Therefore, the MPS is allowed to undergo Cumulative Shortage Process (CSP). The basic idea is that accumulating random amount of shortages due to successive attritions leads to the breakdown of the system when the total shortage crosses a random threshold level. The breakdown point or the threshold can also be interpreted as that point at which immediate recruitment is necessitated.

The shortage of MPS depends on individual propensity to leave the organization, which in turn depends on various factors as discussed before. Such models have been discussed by Grinold and Marshall [5], Bartholomew and Forbes [2]. For statistical approach one may refer to Bartholomew [1]. Lesson [6] has given methods to compute shortages (Resignations, Dismissals, Deaths) and promotion intensities, which produce the proportions corresponding to some descried planning probpsals. Markovian models are designed for shortage and promotion in MPS by Vassiliou [13]. Subramanian. V [11] has made an attempt to provide optimal policy for recruitment, training, promotion and shortages in manpower planning models with special provisions such as time bound promotions, cost of training and voluntary retirement schemes.

Esary et al. [3] have discussed that any component or decice, when exposed to shocks which cause damage to the device or system, is likely to fail when the total accumulated damage exceeds a level called threshold. R. Ramanarayanan and G.Sankaranarayanan [10], in their paper "On Correlated Life and Repair times" have studied the same using Gaver D.P. [4]. Linton D.G. and Saw J.G. [7] hav done Reliability analysis of k-out-of-n : F system. In manpower planning, threshold having SCBZ (setting the clock back to zero) property has been discussed and the expected time to recruitment has been found by Sathiyamoorthy R. and Parthasarathy. S. [12]. For MPS having threshold that follows Exponential distribution, expected time to recruitment and variance have been obtained by S. Parthasarathy and R. Vinoth [9]. Probabilistic Analysis of Time to Recruit and Recruitment Time in Manpower System with two groups by S.Mythilli, R.Ramanarayanan [8].

In this paper, two groups of the MPS are exposed to shortage processes.

Group A consists of employees of MPS other than top level management executives. Group B consists of top level management executives. We assume that after the threshold of MPS recruitment takes places one by one. Here group A is exposed to CSP and group B has Erlang Phase 2 life distribution. We find the joint Laplace Transforms of Time to recruit, Recruitment time, sales time and their expectation. Numerical cases are presented.

\subsection{Model Description}

II. Model - 1

1. MPS has two groups A and B. Group A has employees of MPS other than top level management executives. Group B consists of top level management executives. Group A is exposed to CSP and group B has Erlang distribution of order 2 with parameter $\lambda$. The time to breakdown of MPS is $T=\min \left\{T_{1}, T_{2}\right\}$ where $T_{1}$ and $T_{2}$ 
are the times to breakdown of group A and B respectively. We assume that the recruitment time $\mathrm{R}$ of a shortage is independent of the shortage magnitude.

2. Shortages occur in group $A$ in accordance with their occurrence time distribution $F(x)$ and $\int_{0}^{\infty} x d F(x)<\infty$.

3. $\quad \mathrm{N}$ is the random number of shortages required for breakdown of group $\mathrm{A}$. The group survives $\mathrm{k}$ shortages with an arbitrary but known probability $\mathrm{P}_{\mathrm{k}}(=\mathrm{P}(\mathrm{N}>\mathrm{k}))$ for $\mathrm{k}=0,1,2, \ldots,\left\{\mathrm{P}_{\mathrm{k}}\right\}$ is assumed to be a decreasing sequence of numbers in the unit interval subject only to $\sum_{k=0}^{\infty} P_{k}<\infty, \quad P_{0}=1$.

4. Let $\mathrm{p}_{\mathrm{k}}=\left(\mathrm{P}_{\mathrm{k}-1}-\mathrm{P}_{\mathrm{k}}\right)$, the probability that group $\mathrm{A}$ has a breakdown on the $\mathrm{k}^{\text {th }}$ shortage, be given by the generating function $\emptyset(S), 0 \leq S \leq 1$. $\quad \mathrm{T}_{2}$ has an Erlang Phase $\mathrm{E}_{2}$ distribution with parameter $\mu$.

5. At time 0 , the MPS comprising of the two groups A and B is free of shortage due to manpower loss and put into operation. Groups A and B function independently during time to recruit.

6. When the breakdown of MPS occurs, recruitment begins immediately. Productive work does not happen in group B when the system breakdown occurs due to group A. When it occurs due to group B, productive work does not happen in group A and recruitment takes place for group A.

7. Recruitment time of the $\mathrm{i}^{\text {th }}$ shortage is $\mathrm{R}_{\mathrm{i}} \cdot \mathrm{R}_{\mathrm{i}}$ 's are independent and identically distributed r.v. with distribution function $\mathrm{R}(\mathrm{y})$, independent of the shortage magnitudes. Recruitment time for group $\mathrm{B}$ is assumed to be $\mathrm{R}$ independent of $\mathrm{R}_{\mathrm{i}}$ 's but with distribution function $\mathrm{R}(\mathrm{y}), \int_{0}^{\infty} y d R(y)<\infty$.

8. $\mathrm{K}$ is the number of projects completed or project produced before the breakdown of MPS.

9. Sales Time of each product $\mathrm{S}$ whose distribution function is $\mathrm{G}(\mathrm{z})$ and produced are sold one by one.

\subsection{Analysis}

As per additive recruitment policy, recruitments are done for all the shortages one at a time after time T. $R_{T}=R_{1}+R_{2}+\ldots+R_{i}$ where $i$ is the number of shortages occurred during $T$. When the MPS breakdown occurs due to group $A$, we have to recruit for $N$ shortages. Hence, $R_{T}=R_{1}+R_{2}+\ldots+R_{N}$.

In the other case, when the MPS breakdown occurs due to group $B$, we have $R_{T}=R_{1}+R_{2}+\ldots+$ $R_{n+1}$ as we have to rejuvenate group $B$ also with the $n(<N)$ shortages that occurred in group $A$ during T.Considering also the numbr of products produced and its sales time we find the joint distribution of $T, R_{T}$ , $\mathrm{K}$ and $\mathrm{S}_{\mathrm{K}}$ is given as

$$
\begin{aligned}
P\left(T \leq x, R_{T} \leq y, K=k, S_{K} \leq z\right) & =\sum_{n=1}^{\infty} p_{n} \int_{0}^{x}\left(e^{-\lambda u}+\lambda u e^{-\lambda u}\right) R_{n}(y) e^{-\mu u} \frac{(\mu u)^{k}}{k !} d F_{n}(u) G_{k}(z)+ \\
& \sum_{n=0}^{\infty} P_{n} R_{n+1}(y) \int_{0}^{x} \lambda(\lambda u) e^{-\lambda u}\left[F_{n}(u)-F_{n+1}(u)\right] d u e^{-\mu x} \frac{(\mu x)^{k}}{k !} G_{k}(z)
\end{aligned}
$$

Here $R_{n}(y)$ and $F_{n}(u)$ are the $n$ fold convolutions of $R(y)$ and $F(u)$ respectively.

The joint probability density function is given by

$$
\begin{aligned}
\frac{\partial}{\partial x} \frac{\partial}{\partial y} \frac{\partial}{\partial z} f(x, y, k, z)= & \sum_{n=1}^{\infty} p_{n}\left(e^{-\lambda x}+\lambda x e^{-\lambda x}\right) f_{n}(x) e^{-\mu x} \frac{(\mu x)^{k}}{k !} g_{k}(z) r_{n}(y)+ \\
& \sum_{n=0}^{\infty} P_{n} r_{n+1}(y) \lambda(\lambda x) e^{-\lambda x}\left[F_{n}(x)-F_{n+1}(x)\right] e^{-\mu x} \frac{(\mu x)^{k}}{k !} g_{k}(z)
\end{aligned}
$$

The term under the first summation symbol of the right side of (1) is the joint probability that the MPS breakdown occurs due to group $\mathrm{A}$ on the $\mathrm{n}^{\text {th }}$ shortage during $(0, \mathrm{x})$ and $\sum_{i=1}^{n} R_{i} \leq y$. The term under the second summation symbol is the joint probability that MPS breakdown occurs due to group B during $(0, \mathrm{x})$, group A survives the $\mathrm{n}$ shortages occurred to it before threshold level and $\sum_{i=1}^{n+1} R_{i} \leq y$.

Applying the Laplace-Stiljies Transform in equation(1), we get

$$
E\left[e^{-\varepsilon T} e^{-\eta R_{T}} \theta^{K} e^{-\chi S_{K}}\right]=\sum_{k=0}^{\infty} \int_{0}^{\infty} \int_{0}^{\infty} \int_{0}^{\infty} f(x, y, n, z) e^{-\varepsilon x} e^{-\eta y} \theta^{k} e^{-\chi z} d x d y d z
$$




$$
=\sum_{k=0}^{\infty} \int_{0}^{\infty} \int_{0}^{\infty} \int_{0}^{\infty}\left[\begin{array}{l}
\sum_{n=1}^{\infty} p_{n}\left(e^{-\lambda x}+\lambda x e^{-\lambda x}\right) f_{n}(x) e^{-\mu x} \frac{(\mu x)^{k}}{k !} g_{k}(z) r_{n}(y)+ \\
\sum_{n=0}^{\infty} P_{n} r_{n+1}(y) \lambda(\lambda x) e^{-\lambda x}\left[F_{n}(x)-F_{n+1}(x)\right] e^{-\mu x} \frac{(\mu x)^{k}}{k !} g_{k}(z)
\end{array}\right] e^{-\varepsilon x} e^{-\eta y} \theta^{k} e^{-\chi z} d x d y d z
$$

On simplication, we get

$$
\begin{aligned}
E\left[e^{-\varepsilon T} e^{-\eta R_{T}} T_{\theta} e^{-\chi S_{K}}\right] & =\phi\left(r^{*}(\eta) f^{*}(A)\right)-\lambda \frac{d}{d A}\left[\phi\left(r^{*}(\eta) f^{*}(A)\right)\right]+ \\
& \frac{\lambda^{2}}{(A)^{2}} r^{*}(\eta)\left(1-f^{*}(A)\right)\left(\frac{1-\phi\left(r^{*}(n) f^{*}(A)\right.}{1-r^{*}(n) f^{*}(A)}\right)-\frac{\lambda^{2}}{A} \mathrm{r}^{*}(\eta) \frac{d}{d A}\left[\left(1-f^{*}(A)\right)\left(\frac{1-\phi\left(r^{*}(n) f^{*}(A)\right)}{1-r^{*}(n) f^{*}(A)}\right)\right]
\end{aligned}
$$

Here $\mathrm{A}=\varepsilon+\lambda+\mu-\mu \theta g^{*}(\chi)$

Here * denotes Laplace-Stieltjes transform.

Using differentiation of equation (3), we get

$$
E[T]=\frac{2\left(1-\phi\left(f^{*}(\lambda)\right)\right)}{\lambda}+\phi^{\prime}\left(f^{*}(\lambda)\right) f^{* \prime}(\lambda)
$$

Using differentiation in equation(3), we get

$$
E\left[R_{T}\right]=\left(\frac{1-\phi\left(f^{*}(\lambda)\right)}{1-f^{*}(\lambda)}-\lambda \frac{d}{d \lambda}\left(\frac{1-\phi\left(f^{*}(\lambda)\right)}{1-f^{*}(\lambda)}\right)\right) \mathrm{E}\left[R_{T_{1}}\right]
$$

Assume that $\mathrm{f}($.$) as exponential density function with parameter \delta$

Using differentiation in equation(3), we get

$$
E\left[S_{K}\right]=\mu \mathrm{E}[\mathrm{T}] \mathrm{E}\left[\mathrm{S}_{1}\right]
$$

and

$$
\mathrm{E}[K]=\mu \mathrm{E}[T]
$$

\begin{tabular}{|c|c|c|c|c|c|c|}
\hline$\frac{\delta \rightarrow}{\lambda}$ & 10 & 20 & 30 & 40 & 50 & 60 \\
\hline 5 & 0.1611 & 0.0903 & 0.0624 & 0.0476 & 0.0385 & 0.0323 \\
\hline 10 & 0.1281 & 0.0806 & 0.0580 & 0.0452 & 0.0369 & 0.0312 \\
\hline 15 & 0.1042 & 0.0717 & 0.0537 & 0.0427 & 0.0353 & 0.0301 \\
\hline 20 & 0.0870 & 0.0641 & 0.0497 & 0.0403 & 0.0338 & 0.0290 \\
\hline 25 & 0.0743 & 0.0576 & 0.0460 & 0.0380 & 0.0322 & 0.0279 \\
\hline 30 & 0.0646 & 0.0521 & 0.0427 & 0.0358 & 0.0307 & 0.0269 \\
\hline
\end{tabular}

\section{Numerical Illustration}

We assume that $\mathrm{p}_{1}=\frac{1}{4}, \mathrm{p}_{2}=\frac{1}{4}, \mathrm{p}_{3}=\frac{1}{2}$ and $E\left[R_{T_{1}}\right]=20$, here $\phi(\mathrm{r})=\sum_{i=0}^{\infty} p_{i} r^{i}$

For the different values of $\delta$ ( say $\delta=10,20,30,40,50,60$ ) and the varying parameter is $\lambda=5,10,15,20,25,30$ in the equation (5) and (6), we get

Table of E[T] 


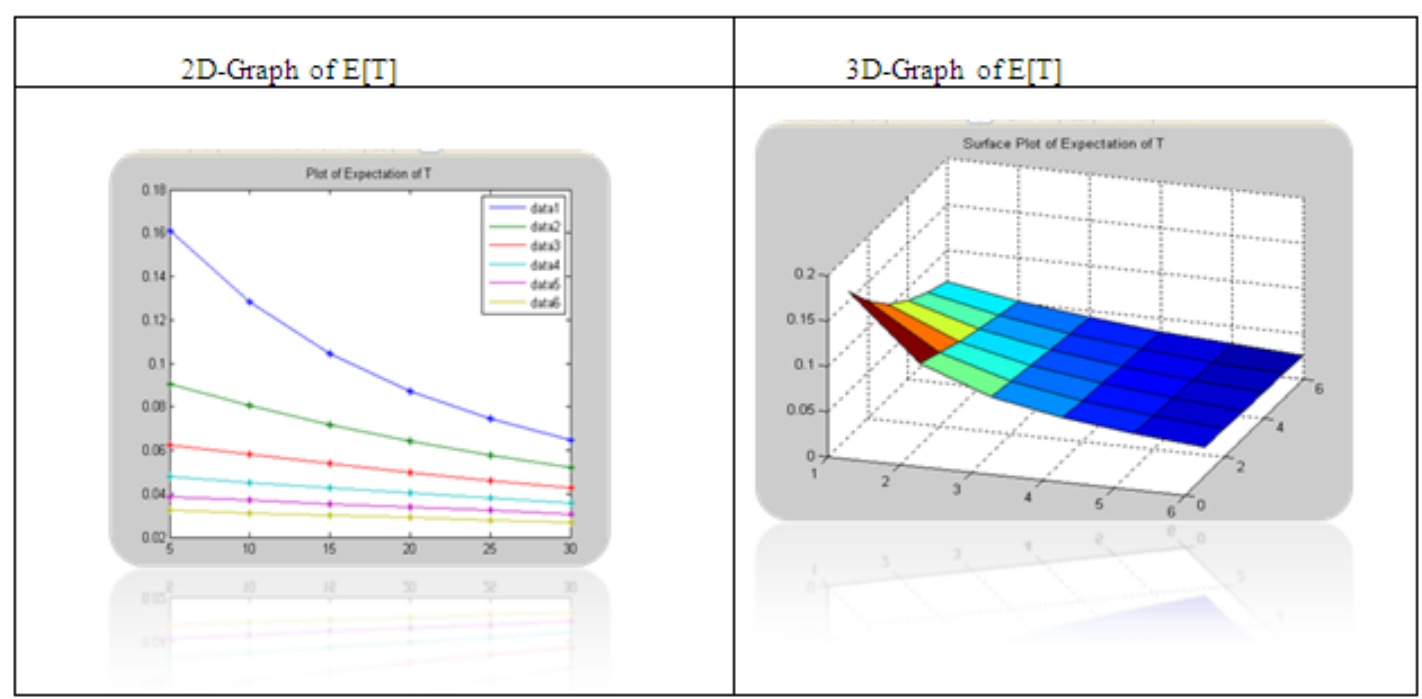

From the above table, as the value of $\delta$ increases, $E[T]$ decreases and the value of $\lambda$ increases, $E[T]$ is also decreases.

Table of $\mathrm{E}\left[\mathrm{R}_{\mathrm{T}}\right]$

\begin{tabular}{|l|c|c|c|c|c|c|}
\hline$\frac{\delta \rightarrow}{\lambda}$ & 10 & 20 & 30 & 40 & 50 & 60 \\
\hline 5 & & & & & & \\
\hline 10 & 30.9259 & 26.4400 & 24.5335 & 23.4911 & 22.8362 & 22.3873 \\
\hline 15 & 36.2500 & 30.9259 & 28.1250 & 26.4400 & 25.3241 & 24.5335 \\
\hline 20 & 39.0800 & 34.0379 & 30.9259 & 28.8918 & 27.4761 & 26.4400 \\
\hline 25 & 40.7407 & 36.2500 & 33.1200 & 30.9259 & 29.3294 & 28.1250 \\
\hline 30 & 41.7930 & 37.8669 & 34.8573 & 32.6195 & 30.9259 & 29.6123 \\
\hline
\end{tabular}

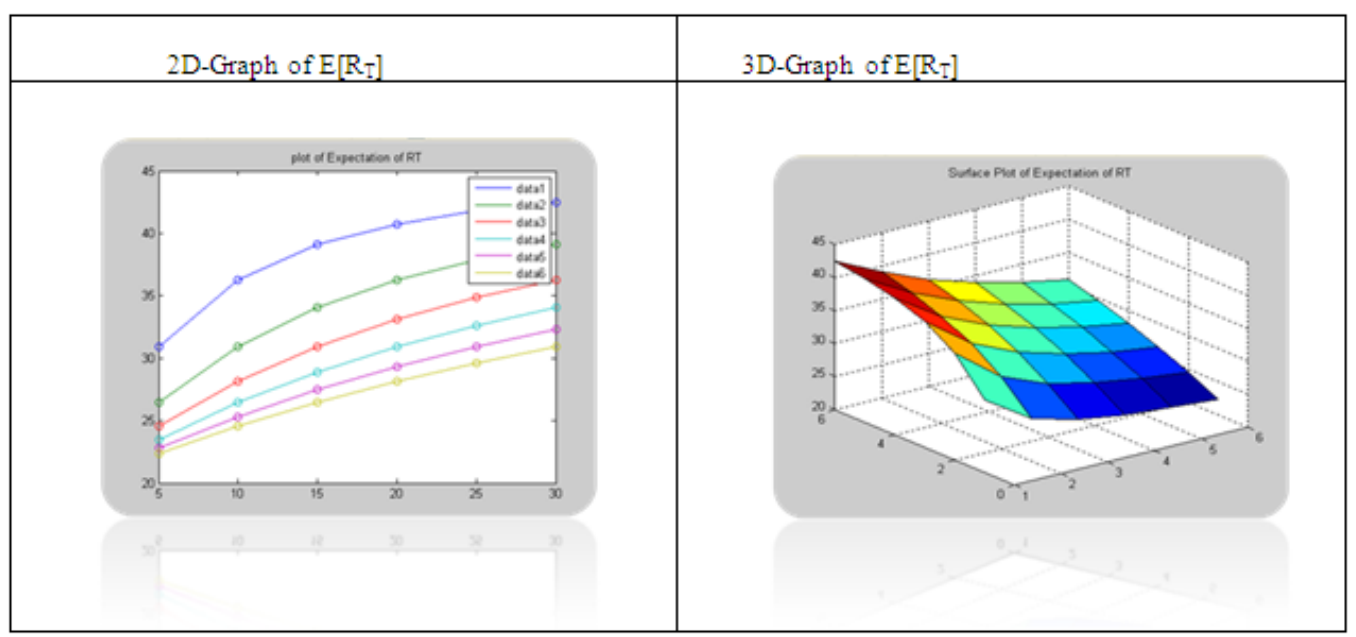

From the above table, as the value of $\delta$ increases, $E\left[R_{T}\right]$ decreases and the value of $\lambda$ increases, $E\left[R_{T}\right]$ increases.

Table of E[K]

\begin{tabular}{|l|l|l|l|l|l|l|}
\hline$\frac{\delta \rightarrow}{\lambda}$ & 10 & 20 & 30 & 40 & 50 & 60 \\
\hline 5 & & & & & & \\
\hline 10 & 0.3222 & 0.1806 & 0.1248 & 0.0952 & 0.0770 & 0.0646 \\
\hline 15 & 0.2562 & 0.1611 & 0.1160 & 0.0903 & 0.07738 & 0.0624 \\
\hline 20 & 0.2085 & 0.1434 & 0.1074 & 0.0854 & 0.0707 & 0.0602 \\
\hline 25 & 0.1741 & 0.1281 & 0.0994 & 0.0806 & 0.0675 & 0.0580 \\
\hline 30 & 0.1486 & 0.1152 & 0.0920 & 0.0760 & 0.0644 & 0.0558 \\
\hline
\end{tabular}


From the above table, as the value of $\delta$ increases, $\mathrm{E}[\mathrm{K}]$ decreases and the value of $\lambda$ increases, $\mathrm{E}[\mathrm{K}]$ decreases.

Table of $\mathbf{E}\left[\mathbf{S}_{\mathbf{K}}\right]$

\begin{tabular}{|l|c|c|c|c|c|c|}
\hline$\frac{\delta \rightarrow}{\lambda}$ & 10 & 20 & 30 & 40 & 50 & 60 \\
\hline 5 & & & & & & \\
\hline 10 & 0.6444 & 0.3613 & 0.2496 & 0.1905 & 0.1535 & 0.1291 \\
\hline 15 & 0.4170 & 0.3222 & 0.2320 & 0.1806 & 0.1477 & 0.1248 \\
\hline 20 & 0.3481 & 0.2568 & 0.2148 & 0.1707 & 0.1413 & 0.1204 \\
\hline 25 & 0.2973 & 0.2304 & 0.1987 & 0.1611 & 0.1350 & 0.1160 \\
\hline 30 & 0.2586 & 0.2085 & 0.1708 & 0.1434 & 0.1230 & 0.1074 \\
\hline
\end{tabular}

From the above table, as the value of $\delta$ increases, $E\left[S_{K}\right]$ decreases and the value of $\lambda$ increases, $E\left[S_{K}\right]$ decreases.

\section{Reference}

[1]. D.J. Bartholonew, The statistical approach to manpower planning, Statistician, 20 (1971), 3-26

[2]. D.J. Bartholomew, A.F. Forbes, Statistical Techniques for Manpower Planning, John Wiley and

[4]. D.P. Gaver, Point Process Problems in Reliability Stochastic Point Processes (Ed. P.A.W. Lewis). Wiley-Interscience, New York (1972), 774-800

[5]. R.C. Grinold, K.J. Marshall, Manpower Planning Models, New York (1977).

[6]. G.W. Lesson, Wastage and promotion in desired manpower structures, J. Opl. Res. Soc., 33 (1982), $433-442$

[7]. D.G. Linton, J.G. Saw, Reliability analysis of the k - out - of - n ; F system, IEEE Trans, Rel. R., 23 (1974), 97-103

[8]. S. Mythili, R. Ramanarayanan, Probabilistic Analysis of Time to Recruit and Recruitment Time in Manpower System with two groups, Applied Mathematical Sciences, Vol.7,2013 no.7, 327-334

[9]. S. Parthasarathy, R. Vinoth., Determination of expected time to recruitment in manpower planning, Recent Research in Science and Technology, 1, No. 3 (2009), 147-150.

[10]. R. Ramanarayanan, G. Sankaranarayanan, On correlated life and repair times, Math. Operations for sch. Statist., Ser. Optimization, 8, No. 1 (1977)

[11]. V. Subramanian, Optimum promotion rate in manpower models, International Journal of Management and Systems, 12 , No. 2 (1996), 179-184.

[12]. R. Sathiyamoorthi, S. Parthasarathy, On the expected time to recruitment when threshold distribution has SCBZ property, IJMS, 19, No. 3 (2003), 233-240

[13]. P.C.G. Vassiliou, A higher order markovian model for prediction of wastage in manpower system, Operat. Res. Quart., 27 (1976), 59-76 